\title{
Gravitino dark matter and a high reheating temperature
}

\section{Krzysztof Turzyński*}

Institute of Theoretical Physics, Warsaw University, Hoża 69, 00-681, Warsaw, Poland

\section{Marek Olechowski}

Institute of Theoretical Physics, Warsaw University, Hoża 69, 00-681, Warsaw, Poland

\section{Stefan Pokorski}

Institute of Theoretical Physics, Warsaw University, Hoża 69, 00-681, Warsaw, Poland

\section{James D. Wells}

CERN Theory Group (PH-TH), CH-1211 Geneva 23, Switzerland and MCTP, University of Michigan, Ann Arbor, MI 48109, USA

\begin{abstract}
Supersymmetry breaking mediated by gauge interactions is regarded an attractive option due to the lack of new sources of flavor changing neutral currents other than those already present in the Standard Model. In models with gauge mediated supersymmetry breaking (GMSB), the dark matter particle is the gravitino. It is produced both thermally in scatterings in the hot plasma and non-thermally from decays of the next to lightest supersymmetric particle (NLSP). If the NLSP is sufficiently abundant during Big Bang Nucleosynthesis (BBN), these decays can alter the abundances of light elements. This, in turn, gives constraints on the gravitino mass and, for the observed dark matter abundance, on the reheating temperature of the Universe. Since sufficiently high reheating temperature is crucial for thermal leptogenesis, one obtains constraints on viable models with GMSB, consistent with thermal leptogenesis. We study the interplay of these constraints within a recently introduced and interesting class of models with GMSB, known as models with general gauge mediation (GGM). We study the possibility that the NLSP is a stau or a sneutrino in these models. We determine whether it is possible to achieve 'compressed' spectra of supersymmetric particles in which gluino is not much heavier than the NLSP, which alleviates the constraints imposed by requiring successful thermal leptogenesis. We study regions of the parameter space of models with GGM and identify regions in which one achieves radiative breaking of electroweak symmetry, satisfies the higgs mass bounds, gravitinos make up the observed amount of dark matter and the reheating temperature is high enough for leptogenesis.
\end{abstract}

European Physical Society Europhysics Conference on High Energy Physics, EPS-HEP 2009,

July 16 - 222009

Krakow, Poland

\footnotetext{
*Speaker.
} 
The question of reconciling supersymmetric dark matter scenarios with the standard evolution of the Universe from very high temperatures was raised long ago [1] and addressed by many authors since. In scenarios with stable gravitinos, such as GMSB [2], it is generically dificult to reach high reheating temperature while maintaining consistency with the BBN bounds. In this case, the decay products of the NLSP threaten to alter BBN. Further, if the gravitino is the only constituent of dark matter, which is the case considered in this paper, its cosmological abundance is fixed to be $\Omega_{\tilde{G}} h^{2}=$ $0.110 \pm 0.006$ [3]. Gravitinos can be thermally produced in the post-inflationary universe, with the abundance proportional to the reheating temperature, $\Omega_{\tilde{G}}^{\mathrm{TP}} h^{2} \propto T_{\mathrm{R}}$, and also proportional to a factor depending on the precise superpartner spectrum. Requirements on $\Omega_{\tilde{G}}$ turn into requirements on $T_{\mathrm{R}}$ for a given superpartner spectrum. This, in turn, leads to strong limits on the reheating temperature $T_{R}$ [4], which may be in conflict with the higher temperatures required for thermal leptogenesis [5]. The goal of this note is to identify the mass spectra that allow for maximal $T_{\mathrm{R}}$ and to describe models of GMSB predicting such mass pattern, following [6].

Very generally, the BBN bounds require that unstable relics previously present in the Universe, decay with lifetimes smaller than $100 \mathrm{~s}$, unless the abundance of these particles is very small or only a tiny fraction of these particles decay with energetic hadrons in the final state [7]. Among the MSSM particles, the latter condition is satisfied by the lightest sneutrino or the lightest stau, hence its parameter space allows for $\tau_{\mathrm{NLSP}}>100 \mathrm{~s}$. The cosmological constraints on late neutrino injection have been worked out [8], providing bounds on decaying sneutrino NLSP. It has been found that sneutrinos with masses smaller than about $330 \mathrm{GeV}$ evade the BBN constraints. Such light sneutrinos are mostly constrained by the requirement that large scale structure formation is not too much affected by free-streaming gravitinos produced in the sneutrino decays [10]. Since the lighter the sneutrino the higher is the reachable reheating temperature, we shall restrict our considerations to a sneutrino in the mass range between 200 and $330 \mathrm{GeV}$. For stau NLSP with masses less than $1 \mathrm{TeV}$, the main $\mathrm{BBN}$ constraint is that of a correct primordial ${ }^{6} \mathrm{Li}$ abundance coming from its catalyzed production [9], ${ }^{6} \mathrm{Li} / \mathrm{H}<6 \times 10^{-11}$.

We are now in a position to calculate the maximal reheating temperature for the sneutrino and stau NLSP. We have $\Omega_{\tilde{G}} h^{2}=\Omega_{\tilde{G}}^{\mathrm{TP}} h^{2}+\left(m_{3 / 2} / m_{\mathrm{NLSP}}\right) \Omega_{\mathrm{NLSP}} h^{2}$, where $\Omega_{\mathrm{NLSP}}$ is the thermal relic abundance that the NLSP would have had, had it not decayed. We calculate the value of this parameter with the micrOMEGAs. 2.2 code [11], assuming that only the NLSP is light. $\Omega_{\tilde{G}}^{\mathrm{TP}} h^{2}$ gives the abundance of thermally produced gravitinos [12], conveniently parametrized as [6]:

$$
\Omega_{\tilde{G}}^{\mathrm{TP}} h^{2}=\left(\frac{T_{\mathrm{R}}}{10^{9} \mathrm{GeV}}\right)\left(\frac{m_{\mathrm{NLSP}}}{300 \mathrm{GeV}}\right)\left[7.4 \times 10^{-6} \frac{\frac{m_{3 / 2}}{1 \mathrm{GeV}}}{\frac{m_{\mathrm{NLSP}}}{300 \mathrm{GeV}}}+\frac{\frac{m_{\mathrm{NLSP}}}{300 \mathrm{GeV}}}{\frac{m_{3} / 2}{1 \mathrm{GeV}}} \sum_{r} \gamma_{r}\left(\frac{M_{r}}{m_{\mathrm{NLSP}}}\right)^{2}\right]
$$

where $r=1,2,3$ corresponds to gauge groups $U(1)_{Y}, S U(2)_{L}$ and $S U(3)_{C}$, respectively, $M_{r}$ denote physical gaugino masses and the coefficients $\gamma_{r}$ depend on the ratios of the gauge couplings at the reheating scale and the scale of the physical gaugino masses. The values of $\gamma_{r}$ can be evaluated for $T_{\mathrm{R}}=10^{9}\left(10^{7}\right) \mathrm{GeV}$ as $\gamma_{3}=0.48-0.56(0.62-0.74), \gamma_{2}=0.57(0.54), \gamma_{1}=0.22(0.17)$, where the range for $\gamma_{3}$ corresponds to the gluino masses ranging from 200 to $900 \mathrm{GeV}$. Obviously, for fixed value of $\Omega_{\tilde{G}}^{\mathrm{TP}} h^{2}$ dominating $\Omega_{\tilde{G}} h^{2}$, the reheating temperature is maximal if the masses of the NLSP and of the three gauginos are degenerate at the low scale (see also [13]).

In general gauge mediation (GGM) [14], the soft masses at the gauge mediation scale $Q$ are 

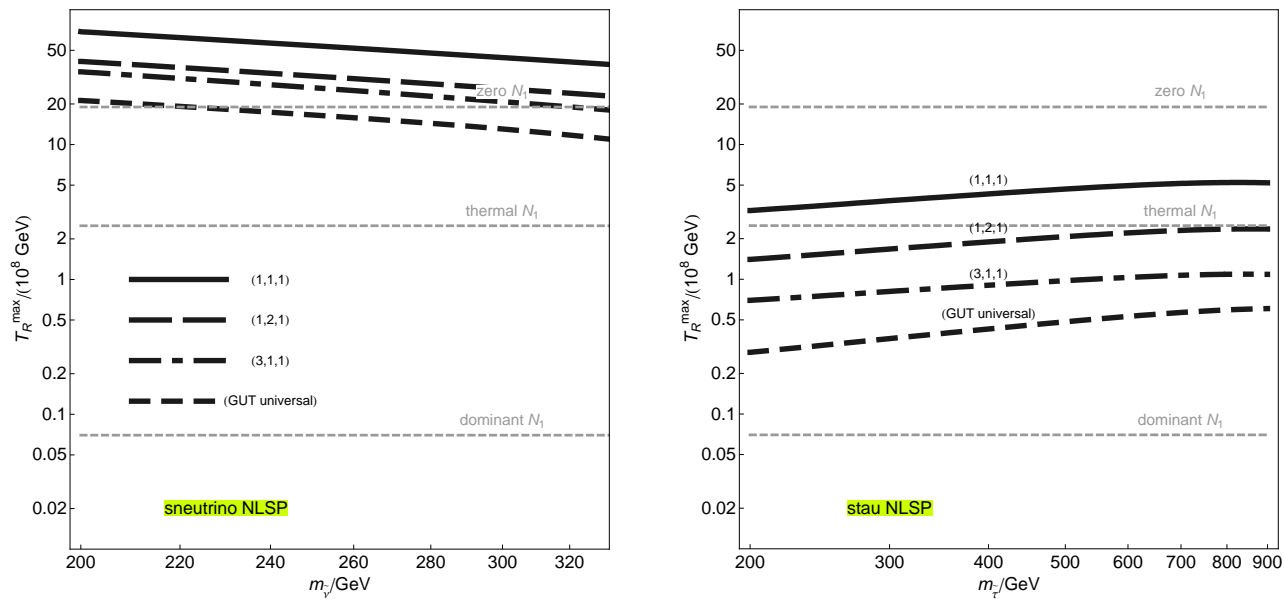

Figure 1: The maximal reheating temperature for the sneutrino (left) and the stau NLSP (right) for four mass patterns of the gauginos at the low-scale $\left(M_{3} / m_{\mathrm{NLSP}}, M_{2} / m_{\mathrm{NLSP}}, M_{1} / m_{\mathrm{NLSP}}\right)=(1,1,1),(1,2,1),(3,1,1)$, and (GUT universal). They correspond, respectively, to solid, dash-dotted, long-dashed, and short-dashed lines. 'Dominant', 'zero' and 'thermal' $N_{1}$ lines correspond to lower limits of $T_{\mathrm{R}}$ needed for leptogenesis given various initial conditions, see [5].

given by $\tilde{M}_{r}=\left(g_{r}^{2} /\left(16 \pi^{2}\right)\right) \Lambda_{r}$ and $\tilde{m}_{s}^{2}=2 \sum_{r=1}^{3}\left(g_{r}^{2} /\left(16 \pi^{2}\right)\right)^{2} C_{r}^{(s)} \kappa_{r} \Lambda_{r}^{2}$. The $a$ priori unknown degrees of freedom in the model are parametrized by $\Lambda_{r}$ and $\kappa_{r}$.

To a good approximation we can identify the lighter stau with the right stau. Imposing the bound $m_{E}^{2}<M_{1}^{2}$, we find $\kappa_{1}<0.089$ for $Q=10^{15} \mathrm{GeV}$ or, equivalently, $\tilde{m}_{E}^{2}<0.1 \tilde{M}_{1}^{2}$ at the high scale. Since in gauge mediation models $\tilde{m}_{E}^{2}>0$, we also have $M_{1}^{2}>m_{E}^{2}>0.6 M_{1}^{2}$. Thus, the low energy masses of stau and bino are almost degenerate, as a result of the RG evolution itself With $Q=10^{14} \mathrm{GeV}$, we obtain a slightly weaker bound $\kappa_{1}<0.13$. In the other case, $m_{L}^{2}<m_{E}^{2}$, which can give sneutrino NLSP, the following three conditions are relevant for constraining the parameter space: $m_{L}^{2}<\left\{m_{E}^{2}, M_{1}^{2}, M_{2}^{2}\right\}$, giving the bounds $\kappa_{1}<0.24-1.8 \kappa_{2}$ and $\kappa_{2}<0.09$. In particular, these bounds imply that $\kappa_{1}<0.24$, and that the bino and wino physical masses must be in the range $0.6 M_{1}<M_{2}<1.2 M_{1}$. For sufficiently large left-right mass splitting compared to the left-right mass mixing term of the slepton mass matrix we get a sneutrino NLSP. With $\mu=1000 \mathrm{GeV}$ and $\tan \beta=10$ this possibility requires a minimal splitting between $\sqrt{m_{E}^{2}}$ and $\sqrt{m_{L}^{2}}$ of about $100 \mathrm{GeV}$.

By tuning $\Lambda_{2}$ and $\Lambda_{3}$ against $\Lambda_{1}$, we can achieve the gluino and wino masses degenerate with the sneutrino/stau NLSP mass. Then we have freedom to choose $\kappa_{3}$ and the absolute scale of the GGM model given by $\Lambda_{1}$ to ensure that the electroweak symmetry is broken, the Higgsino is heavier than the NLSP and that the stops are heavy enough to satisfy the Higgs boson mass bound.

To summarize, having identified the mass patterns of the MSSM that allow for maximal reheating temperature, we found the parameter sets of the GGM models leading to such mass patterns. Interestingly, these parameter sets arise in rather simple models, with messenger belonging to $\mathbf{4 0}+\overline{\mathbf{4 0}}, 3 \times \mathbf{2 4}$ or $\mathbf{7 5}$ representations of the unified gauge group $S U(5)$.

Acknowledgements. This work was partially supported by TOK Project MTKD-CT-2005029466, the EC 6th Framework Programme MRTN-CT-2006-035863 and the U.S. Department of Energy. KT is partially supported by the Foundation for Polish Science through its programme 
Homing.

\section{References}

[1] J. R. Ellis, A. D. Linde and D. V. Nanopoulos, Phys. Lett. B 118 (1982) 59; M. Y. Khlopov and A. D. Linde, Phys. Lett. B 138 (1984) 265; J. R. Ellis, J. E. Kim and D. V. Nanopoulos, Phys. Lett. B 145 (1984) 181; J. R. Ellis, D. V. Nanopoulos and S. Sarkar, Nucl. Phys. B 259 (1985) 175; F. Balestra et al., Nuovo Cim. A 92 (1986) 139; M. Kawasaki and T. Moroi, Prog. Theor. Phys. 93 (1995) 879 [arXiv:hep-ph/9403364]; J. R. Ellis, D. V. Nanopoulos, K. A. Olive and S. J. Rey, Astropart. Phys. 4 (1996) 371 [arXiv:hep-ph/9505438]; M. Bolz, A. Brandenburg and W. Buchmuller, Nucl. Phys. B 606 (2001) 518 [Erratum-ibid. B 790 (2008) 336] [arXiv:hep-ph/0012052].

[2] G. F. Giudice and R. Rattazzi, Phys. Rept. 322 (1999) 419 [arXiv:hep-ph/9801271].

[3] J. Dunkley et al. [WMAP Collaboration], Astrophys. J. Suppl. 180 (2009) 306 [arXiv:0803.0586 [astro-ph]].

[4] L. Roszkowski, R. Ruiz de Austri and K. Y. Choi, JHEP 0508 (2005) 080 [arXiv:hep-ph/0408227]; D. G. Cerdeno, K. Y. Choi, K. Jedamzik, L. Roszkowski and R. Ruiz de Austri, JCAP 0606 (2006) 005 [arXiv:hep-ph/0509275]; J. Pradler and F. D. Steffen, Phys. Lett. B 648 (2007) 224 [arXiv:hep-ph/0612291]; K. Y. Choi, L. Roszkowski and R. Ruiz de Austri, JHEP 0804 (2008) 016 [arXiv:0710.3349 [hep-ph]]; F. D. Steffen, Phys. Lett. B 669 (2008) 74 [arXiv:0806.3266 [hep-ph]]; M. Kawasaki, K. Kohri, T. Moroi and A. Yotsuyanagi, Phys. Rev. D 78 (2008) 065011 [arXiv:0804.3745 [hep-ph]].

[5] G. F. Giudice, A. Notari, M. Raidal, A. Riotto and A. Strumia, Nucl. Phys. B 685 (2004) 89 [arXiv:hep-ph/0310123]; S. Antusch and A. M. Teixeira, JCAP 0702 (2007) 024 [arXiv:hep-ph/0611232]; S. Davidson, E. Nardi and Y. Nir, Phys. Rept. 466 (2008) 105 [arXiv:0802.2962 [hep-ph]].

[6] M. Olechowski, S. Pokorski, K. Turzynski and J. D. Wells, arXiv:0908.2502 [hep-ph].

[7] M. Kawasaki, K. Kohri and T. Moroi, Phys. Rev. D 71, 083502 (2005) [arXiv:astro-ph/0408426].

[8] T. Kanzaki, M. Kawasaki, K. Kohri and T. Moroi, Phys. Rev. D 76 (2007) 105017 [arXiv:0705.1200 [hep-ph]]; K. Jedamzik, JCAP 0803, 008 (2008) [arXiv:0710.5153 [hep-ph]].

[9] S. Dimopoulos, D. Eichler, R. Esmailzadeh and G. D. Starkman, Phys. Rev. D 41 (1990) 2388; M. Pospelov, Phys. Rev. Lett. 98 (2007) 231301 [arXiv:hep-ph/0605215].

[10] K. Jedamzik, M. Lemoine and G. Moultaka, JCAP 0607 (2006) 010 [arXiv:astro-ph/0508141].

[11] G. Belanger, F. Boudjema, A. Pukhov and A. Semenov, Comput. Phys. Commun. 176 (2007) 367 [arXiv:hep-ph/0607059]; G. Belanger, F. Boudjema, A. Pukhov and A. Semenov, Comput. Phys. Commun. 180 (2009) 747 [arXiv:0803.2360 [hep-ph]].

[12] J. Pradler and F. D. Steffen, Phys. Rev. D 75 (2007) 023509 [arXiv:hep-ph/0608344]; V. S. Rychkov and A. Strumia, Phys. Rev. D 75 (2007) 075011 [arXiv:hep-ph/0701104].

[13] M. Fujii, M. Ibe and T. Yanagida, Phys. Lett. B 579 (2004) 6 [arXiv:hep-ph/0310142].

[14] P. Meade, N. Seiberg and D. Shih, Prog. Theor. Phys. Suppl. 177 (2009) 143 [arXiv:0801.3278 [hep-ph]]; L. M. Carpenter, M. Dine, G. Festuccia and J. D. Mason, Phys. Rev. D 79 (2009) 035002 [arXiv:0805.2944 [hep-ph]]. 